\title{
A note on the Cornaz-Jost transformation to solve the graph coloring problem
}

\author{
Flavia Bonomo ${ }^{\mathrm{a}, *, 1}$, Monia Giandomenico ${ }^{\mathrm{b}}$, Fabrizio Rossi ${ }^{\mathrm{b}}$ \\ a IMAS-CONICET and Departamento de Computación, FCEN, Universidad de Buenos Aires, Buenos Aires, Argentina \\ b Dipartimento di Informatica, Università di L'Aquila, L'Aquila, Italy
}

\section{A R T I C L E I N F O}

\section{Article history:}

Received 30 January 2013

Received in revised form 30 May 2013

Accepted 31 May 2013

Available online 4 June 2013

Communicated by R. Uehara

\section{Keywords:}

Graph coloring problem

Graph operators

Max-coloring problem

Polynomial-time algorithm

Computational complexity

\begin{abstract}
A B S T R A C T
In this note, we use a reduction by Cornaz and Jost from the graph (max-)coloring problem to the maximum (weighted) stable set problem in order to characterize new graph classes where the graph coloring problem and the more general max-coloring problem can be solved in polynomial time.
\end{abstract}

(c) 2013 Elsevier B.V. All rights reserved.

\section{Introduction}

A stable set of a graph is a subset of pairwise nonadjacent vertices, and a coloring of a graph is a partition of its vertices into nonempty stable sets. The maximum cardinality of a stable set of a graph $G$ is denoted by $\alpha(G)$, and the minimum number of stable sets in a coloring of $G$, called the chromatic number of $G$, is denoted by $\chi(G)$. The graph coloring problem is a basic model for scheduling, frequency assignment, and resource allocation problems. From particular constraints arising in practical settings, more elaborate models of coloring have been defined in the literature.

\footnotetext{
* Corresponding author.

E-mail addresses: fbonomo@dc.uba.ar (F. Bonomo), giandomenico@di.univaq.it (M. Giandomenico), fabrizio.rossi@univaq.it (F. Rossi).

1 Partially supported by Progetto regionale speciale multiasse "Reti per la conoscenza e l'orientamento tecnico-scientifico per lo sviluppo della competitività" - RE.C.O.TE.S.S.C. - POR 2007-2013 - Azione 4 (Italy) and CONICET PIP 112-200901-00178, UBACyT Grant 20020100100980 and ANPCyT PICT-2012-1324 (Argentina).
}

Given a graph $G$ with a nonnegative weight $w$ associated to each vertex $v$, the max-coloring problem consists of finding a coloring of $G$ that minimizes the sum, over all stable sets in the partition, of the maximum weight of a vertex in the set. It has applications in batch scheduling [5,8] and buffer minimization [10].

The graph coloring problem is NP-complete in general, but it can be solved in polynomial time for several classes, being the most prominent the class of perfect graphs [9]. For a compendium of graph classes and the corresponding computational complexity of the coloring problem on them, see [6]. Max-coloring is substantially harder than the graph coloring problem, in particular it is NP-hard in the strong sense in split graphs [5], and so in particular in perfect graphs and in $P_{5}$-free graphs. Restricted to bipartite graphs, it is NP-hard in $P_{8}$-free bipartite graphs but polynomial-time solvable in $P_{5}$-free bipartite graphs [4].

In a recent paper, Cornaz and Jost [3] exhibit a new polynomial-time reduction from the graph coloring problem to the maximum stable set problem. Namely, given a graph $G$ with $n$ vertices and $m$ edges, they construct an auxiliary graph $T(G)$ with $\bar{m}$ vertices such that the set of all stable sets of $T(G)$ is in one-to-one correspondence 
with the set of all colorings of $G$, where $\bar{m}$ is the number of edges of the complement graph $\bar{G}$ of $G$.

In fact, the reduction is more general and applies also to weighted graphs. They reduce the max-coloring problem to the maximum weighted stable set problem.

A maximum weighted stable set of $G$ is a set of pairwise nonadjacent vertices such that the sum of the weights of the vertices in the set is maximum. The maximum weighted stable set problem and its unweighted version are NP-complete in general, and they can be solved in polynomial time on perfect graphs [9] and apple-free graphs [1] and in $O\left(n^{3}\right)$ time on claw-free graphs [7].

A claw is a graph formed by a vertex with three neighbors of degree one. A hole in a graph $G$ is an induced cycle of length at least five. An antihole is the complement of a hole. A hole or antihole is odd if it has an odd number of vertices. Denote by $C_{k}$ the induced cycle of length $k$, and by $P_{k}$ the induced path of $k$ vertices. An apple $A_{k}$ is a graph obtained from a chordless cycle $C_{k}$ of length $k \geqslant 4$ by adding a vertex that has exactly one neighbor on the cycle. If $H$ is a graph, a graph $G$ is called $H$-free if no induced subgraph of $G$ is isomorphic to $H$. If $\mathcal{H}$ is a family of graphs, a graph $G$ is called $\mathcal{H}$-free if no induced subgraph of $G$ is isomorphic to a graph in $\mathcal{H}$.

A clique of a graph is a subset of pairwise adjacent vertices. The maximum cardinality of a clique of $G$ is denoted by $\omega(G)$. A graph $G$ is perfect when $\chi(H)=\omega(H)$ for every induced subgraph $H$ of $G$. Equivalently, a graph is perfect if and only if it contains neither odd holes nor odd antiholes as induced subgraphs [2].

Given a graph $G$, denote by $V(G)$ and $E(G)$ the set of vertices and edges of $G$, respectively. Let $n=|V(G)|$ and $m=|E(G)|$. Denote by $\bar{G}$ the complement of $G$, and by $\bar{m}$ the number of edges of $\bar{G}$. Denote by $L(G)$ the line graph of $G$, that is, the intersection graph of the edges of $G$. A partial subgraph of $G$ is a graph $G^{\prime}$ such that $V\left(G^{\prime}\right) \subseteq$ $V(G)$ and $E\left(G^{\prime}\right) \subseteq E(G)$. A spanning partial subgraph of $G$ is a partial subgraph $G^{\prime}$ such that $V\left(G^{\prime}\right)=V(G)$.

Let $D$ be a simple digraph with vertex set $V(D)$ and arc set $A(D)$. An arc with tail $u$ and head $v$ is denoted by $u v$. The digraph $D$ is called acyclic if it has no directed cycle. Recall that $D$ is acyclic if and only if there is a total ordering $\prec$ on its vertex set such that $u \prec v$ for each arc $u v$. A pair of arcs of $D$ is called a simplicial pair of $D$ if they share the tail and their heads are connected by an arc.

Let $D$ be an acyclic orientation of the complement $\bar{G}$ of a graph $G$. The graph $T(G)$ is obtained from the line graph of $\bar{G}$ by removing all edges between pairs of edges of $\bar{G}$ which are simplicial pairs of arcs in $D$.

Theorem 1. (See [3].) For any graph $G$ and any acyclic orientation of its complement $\bar{G}$, there is a one-to-one correspondence between the set of all colorings of $G$ and the set of all stable sets of $T(G)$. Moreover, $\alpha(T(G))+\chi(G)=|V(G)|$.

Note that $|V(T(G))|=\bar{m}$ and $|E(T(G))|$ is equal to the number of edges of the line graph of $\bar{G}$ minus the number of triangles in $\bar{G}$. Therefore, given an undirected graph $G$, the number of vertices and edges of $T(G)$ does not depend on the order of $V(G)$ from which the orientation of $D$ is derived.
Given a graph $G$ with a nonnegative weight $w$ on its vertex set, denote by $\alpha_{w}(G)$ the weight of a maximum weighted stable set of $G$ with respect to $w$, and by $\chi_{w}(G)$ the value of an optimum max-coloring of $G$ with respect to $w$. For each vertex $a$ of $T(G)$ corresponding to the arc $u v$ of $D$, define the weight $\tilde{w}(a):=w(v)$. The previous theorem can be generalized to weighted graphs in the following way.

Theorem 2. (See [3].) Let $G$ be a graph with a nonnegative weight $w$ on its vertices, and consider an acyclic orientation of $\bar{G}$ given by a nonincreasing ordering of $V(G)$ with respect to $w$. Then $\alpha_{\tilde{w}}(T(G))+\chi_{w}(G)=w(V(G))$.

In this paper, we analyze the transformation $T$. In particular, we try to find classes of graphs $\mathcal{C}$ such that $T(\mathcal{C})$ is a class of graphs where the maximum weighted stable set is polynomial-time solvable. In general, we consider $T(\mathcal{C})$ as the class of graphs $T(G)$ obtained from any acyclic orientation of the complement of a graph $G$ in $\mathcal{C}$ since, for the max-coloring problem, this orientation is given by the weight function. But one of our main results is the characterization of a class of graphs $\mathcal{C}$ in which, for every graph $G$ in $\mathcal{C}$, there exists an orientation of $\bar{G}$ such that $T(G)$ is claw-free. In that class, the coloring problem is polynomial-time solvable, by using any available polynomial-time algorithm for maximum stable set in claw-free graphs. The class obtained is not contained in any previously known class where the coloring problem is polynomial-time solvable, as far as we could check in [6] Moreover, it can be recognized in polynomial time, which makes the result interesting also from a practical point of view.

\section{Main results}

We start by analyzing the pre-image of perfect graphs by the transformation $T$. In what follows we denote with $u v$ the vertex in $V(T(G)$ ) corresponding to the arc $u v$ of $D$.

Proposition 3. If $G$ has an odd hole as induced subgraph then $T(G)$ has a hole of length 5 as induced subgraph, and if $G$ has an odd antihole $\bar{C}_{k}$ as induced subgraph then $T(G)$ has an odd hole $C_{k}$ as induced subgraph.

Proof. If $G$ has $\bar{C}_{k}$ as induced subgraph, then $\bar{G}$ has $C_{k}$ as induced subgraph, and no pair of edges of it is a simplicial pair of arcs in $D$. So $T(G)$ has $C_{k}$ as induced subgraph. In particular, for $k=5$, if $G$ has $\bar{C}_{5}=C_{5}$ as induced subgraph, then $T(G)$ has $C_{5}$ as induced subgraph.

Let $H$ be a subgraph of $G$ inducing an odd hole with vertex set $V(H)=\left\{v_{1}, \ldots, v_{k}\right\}$, where $k \geqslant 7$ and $v_{1}$ is the smallest vertex of $H$ in the vertex ordering defining the orientation of $D$. Then, vertices $\left\{v_{1} v_{5}, v_{1} v_{4}, v_{4} v_{6}\right.$, $\left.v_{3} v_{6}, v_{3} v_{5}\right\}$ induce a hole of length 5 in $T(G)$.

Corollary 4. If $T(G)$ is a perfect graph then $G$ is a perfect graph. That is, $T^{-1}$ (perfect graphs) $\subseteq$ perfect graphs. 


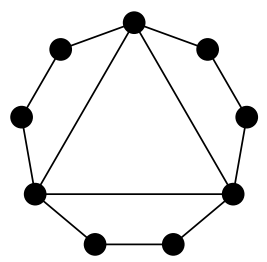

Fig. 1. The complement $\bar{G}$ of a perfect graph $G$ such that $T(G)$ is not perfect, independently of the orientation of $\bar{G}$.

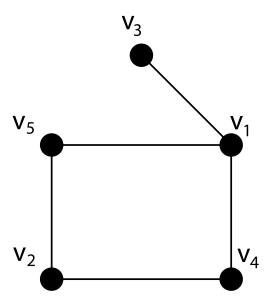

Fig. 2. The apple $A_{4}$.

Note that if $G$ is perfect then $T(G)$ is not necessarily perfect. In particular, there exists a perfect graph $G$ such that $T(G)$ is not perfect, independently of the acyclic orientation of its complement (Fig. 1). So, from the graph coloring point of view, the pre-image of perfect graphs by the transformation $T$ leads to a class of graphs where the problem is already known to be polynomialtime solvable. But it could be of interest to characterize $T^{-1}$ (perfect graphs) from the max-coloring point of view.

Our aim now is to characterize $T^{-1}$ (claw-free graphs), in order to describe a new class in which the max-coloring problem can be solved in polynomial time.

Lemma 5. $T(G)$ is a claw-free graph for every acyclic orientation of $\bar{G}$ if and only if $G$ does not contain a spanning partial subgraph of $A_{4}$ (Fig. 2) as an induced subgraph.

Proof. Suppose first that $G$ contains a spanning partial subgraph $\mathrm{H}$ of $A_{4}$ as induced subgraph. Let us consider an ordering of the vertices of $\bar{G}$ such that $v_{1} \prec v_{2} \prec v_{3} \prec v_{4} \prec$ $v_{5}$, and let $D$ be the digraph obtained from that ordering. Then $v_{3} v_{4}$ and $v_{3} v_{5}$ are a simplicial pair of $D$, so they are nonadjacent in $T(G)$. Besides, $v_{2} v_{3}$ does not form a simplicial pair of $D$ with neither $v_{1} v_{2}, v_{3} v_{4}$ nor $v_{3} v_{5}$, independently of which edges were removed from $A_{4}$ to obtain $H$. So, $\left\{v_{2} v_{3}, v_{1} v_{2}, v_{3} v_{4}, v_{3} v_{5}\right\}$ induces a claw in $T(G)$.
Conversely, suppose that $T(G)$ contains an induced claw, for some ordering of the vertices of $\bar{G}$. Let $a b, c d$, ef and $g h$ be the edges of $\bar{G}$ inducing the claw on $T(G)$, where $c d$, ef and $g h$ form a stable set on $T(G)$ and $a b$ is adjacent to all of them. We will split the proof into two cases: $c d$, ef and $g h$ share the same endpoint of $a b$ (wlog, $c=e=g=a$ ), or two of them share an endpoint with $a b$ and the third one shares the other endpoint with $a b$ (wlog, $c=e=a$ and $g=b$ ). In the first case, each pair of edges in $\{c d, e f, g h\}$ is a simplicial pair, so $d, f$ and $h$ form a triangle in $\bar{G}$, and $\{b, a, d, f, h\}$ induces on $G$ a subgraph of $A_{4}$. In the second case, $\{c d, e f\}$ is a simplicial pair, so $d$ and $f$ are adjacent in $\bar{G}$, and $a \prec d, f$. If $h$ is different from $d$ and $f$, then $\{h, b, a, d, f\}$ induces on $G$ a subgraph of $A_{4}$. So, suppose wlog that $h=d$. In that case, $g h(=b d)$ and $c d(=a d)$ should form a simplicial pair, but this is impossible because $a \prec d$, and this completes the proof of the proposition.

Remark 1. If $T(G)$ is a claw-free graph for some acyclic orientation of $\bar{G}$ then $\alpha(G) \leqslant 4$.

As a direct consequence of the previous lemma, we have the following result.

Theorem 6. $T(G)$ is a claw-free graph for every acyclic orientation of $\bar{G}$ if and only if $G$ does not contain $P_{5}$ or a graph in Fig. 3 as an induced subgraph.

Corollary 7. Given a graph $G$ that does not contain $P_{5}$ or a graph in Fig. 3 as an induced subgraph, and a nonnegative weight on its vertices, the max-coloring problem on $G$ can be solved in $O\left(\bar{m}^{3}\right)$.

We can strength this result for coloring by choosing a clever ordering of the vertices of $G$ in order to obtain an orientation of the graph $\bar{G}$ leading to a claw-free graph $T(G)$, even if we allow to have some induced $P_{5}$ 's in $G$.

Theorem 8. If $G$ does not contain a graph in Fig. 3 as an induced subgraph and there exists an ordering $\prec$ of the vertices of $G$ such that for every induced $P_{5}=v_{1} v_{2} v_{3} v_{4} v_{5}$ of G it holds $v_{3} \prec v_{1}$ and $v_{3} \prec v_{5}$, then the graph $T(G)$, obtained from the acyclic orientation of $\bar{G}$ given by that ordering, is a claw-free graph.

Proof. Let $G$ be a graph and consider an ordering $\prec$ of its vertices. Suppose, by the way of contradiction, that $T(G)$ is
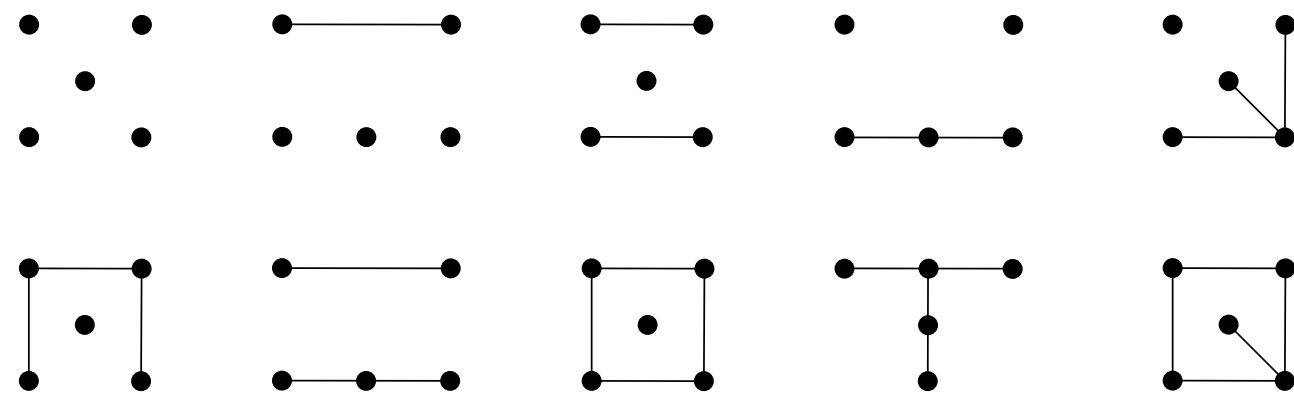

Fig. 3. All spanning partial subgraphs of $A_{4}$ but $P_{5}$.
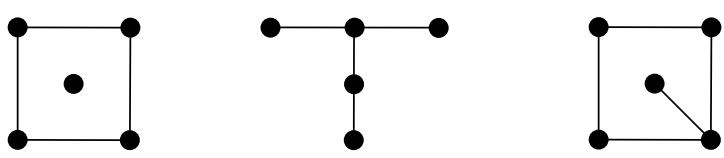
not a claw-free graph. Then, by Proposition 5, $G$ contains either a graph in Fig. 3 or a path $H=v_{1} v_{2} v_{3} v_{4} v_{5}$ as induced subgraph. Since $G$ does not contain a graph in Fig. 3 as an induced subgraph, then the claw in $T(G)$ is formed by the edges of $\bar{H}$. It is easy to check that they induce a claw in $T(G)$ if and only if either $v_{1} \prec v_{3}$ or $v_{5} \prec v_{3}$.

From Theorem 8, one can characterize the following class of graphs in which the coloring problem can be solved in polynomial time.

Corollary 9. Given a graph $G$, it can be checked in polynomial time if $G$ does not contain a graph in Fig. 3 as an induced subgraph and there exists an ordering $\prec$ of the vertices of $G$ such that for every induced $P_{5}=v_{1} v_{2} v_{3} v_{4} v_{5}$ of $G$ it holds $v_{3} \prec v_{1}$ and $v_{3} \prec v_{5}$. In this case, the coloring problem can be solved in polynomial time for $G$. The overall complexity of the algorithm is $O\left(n^{5}+\bar{m}^{3}\right)$.

Proof. It can be checked in $O\left(n^{5}\right)$ time that $G$ does not contain a graph in Fig. 3 as an induced subgraph. Also in $O\left(n^{5}\right)$ time it can be built a digraph $D^{\prime}$ with vertex set $V(G)$ and an oriented arc for each pair of vertices $x, y$ such that there is an induced $P_{5}$ in $G$ such that $x$ is the middle vertex and $y$ is an end vertex of it. Finally, it can be checked in linear time if $D^{\prime}$ is acyclic, and in that case it can be given in linear time a suitable ordering for $V(G)$. Finally, the algorithm to solve the coloring problem consists on building the graph $T(G)$ with respect to that order and, by Theorem 1, solving the stable set problem on it. By Theorem 8, $T(G)$ is a claw-free graph and so, the stable set problem can be solved in $O\left(\bar{m}^{3}\right)$ time [7]

One might be tempted to try to enlarge the class by looking at the pre-image of apple-free graphs by the transformation $T$, since apple-free graphs constitute a superclass of claw-free graphs where the maximum weighted stable set problem can be solved in polynomial time as well. Unfortunately, a computer-aided exhaustive case analysis shows that if, for a given ordering of the vertices of $G, T(G)$ contains a claw as induced subgraph then it also contains an induced $A_{4}$. In other words, $T(G)$ is applefree if and only if it is claw-free. As a consequence, if $T(G)$ is a chordal graph then it is also claw-free, and in particular if $T(G)$ is a tree then it is indeed a path.

\section{References}

[1] A. Brandstädt, V. Lozin, R. Mosca, Independent sets of maximum weight in apple-free graphs, SIAM Journal on Discrete Mathematics 24 (1) (2010) 239-254.

[2] M. Chudnovsky, N. Robertson, P. Seymour, R. Thomas, The strong perfect graph theorem, Annals of Mathematics 164 (1) (2006) 51-229.

[3] D. Cornaz, V. Jost, A one-to-one correspondence between colorings and stable sets, Operations Research Letters 36 (6) (2008) 673-676.

[4] D. de Werra, M. Demange, B. Escoffier, J. Monnot, V. Paschos, Weighted coloring on planar, bipartite and split graphs: Complexity and approximation, Discrete Applied Mathematics 157 (4) (2009) 819-832.

[5] M. Demange, D. de Werra, J. Monnot, V. Paschos, Weighted node coloring: When stable sets are expensive, Lecture Notes in Computer Science 2573 (2002) 114-125.

[6] H. de Ridder, et al., Information system on graph classes and their inclusions (ISGCI), http://www.graphclasses.org.

[7] Y. Faenza, G. Oriolo, G. Stauffer, An algorithmic decomposition of claw-free graphs leading to an $O\left(n^{3}\right)$-algorithm for the weighted stable set problem, in: D. Randall (Ed.), Proceedings of the 22nd Annual ACM-SIAM Symposium on Discrete Algorithms, San Francisco, CA, 2011, pp. 630-646.

[8] G. Finke, V. Jost, M. Queyranne, A. Sebö, Batch processing with interval graph compatibilities between tasks, Discrete Applied Mathematics 156 (5) (2008) 556-568.

[9] M. Grötschel, L. Lovász, A. Schrijver, The ellipsoid method and its consequences in combinatorial optimization, Combinatorica 1 (1981) 169-197.

[10] S. Pemmaraju, R. Raman, K. Varadarajan, Buffer minimization using max-coloring, in: J. Ian Munro (Ed.), Proceedings of the 15th Annual ACM-SIAM Symposium on Discrete Algorithms, New Orleans, LA, 2004, pp. 562-571. 